\title{
Design of a Static Full-Ring Multi-Pinhole Collimator for Brain SPECT
}

\author{
Karen Van Audenhaege, Student Member, IEEE, Roel Van Holen, Member, IEEE, Karel Deprez, \\ Joel S. Karp, Senior Member, IEEE, Scott Metzler, Member, IEEE, and Stefaan Vandenberghe
}

\begin{abstract}
In clinical practice, brain SPECT is mostly performed using a dual-head SPECT scanner with fan-beam or parallel-beam collimators rotating around the patient's head. The resolution of such a system is typically about $6-8 \mathrm{~mm}$, which is rather poor to image the complex structures of the human brain.

We developed a non-rotating multi-pinhole collimator for brain SPECT imaging with a resolution of $4 \mathrm{~mm}$. A full-ring geometry allows for complete transaxial sampling. This enables the use of a stationary collimator. The collimator is a tungsten ring with two rows of pinholes. Each pinhole can individually be opened or closed with shutters. A sequence of shutter movements is performed to obtain an acquisition setup that simulates a rotational movement. The collimator is designed for the LaPET system (a PET detector ring made of $24 \mathrm{LaBr}_{3}$ detectors) and is optimized to maximize the system performance, resulting in a collimator radius of $145 \mathrm{~mm}$ and a pinhole diameter of $2 \mathrm{~mm}$. This system has a sensitivity that is $\mathbf{4}$ times lower than a dual-head system with LEHR parallel-beam collimators. However, the resolution is 2 times better, a trade-off that is supported by Muehllehner [1]. Monte-Carlo simulated projections of a resolution phantom are successfully reconstructed and the resulting image shows that a resolution of $4 \mathrm{~mm}$ is indeed achieved.
\end{abstract}

Index Terms-Imaging, instrumentation, SPECT, system design, collimator

\section{INTRODUCTION}

$\mathbf{S}$ INGLE Photon Emission Computed Tomography (SPECT) is a technique often used in nuclear medecine for the diagnosis of neurological disorders. Tracers such as the Tc-99m labeled HMPAO and ECD are widely used in clinical practice for cerebral perfusion imaging which is used in the evaluation of epilepsy, cerebrovascular disease, trauma and dementia [2].

In clinical practice, brain imaging is often performed with a dual-head SPECT scanner with fan-beam or parallel-beam collimators rotating around the patient's head. The resolution of such a system is typically $6-8 \mathrm{~mm}$, which is rather poor to image the complex structures of the human brain. Therefore, brain imaging is now often performed with Positron Emission

This work was supported by Ghent University (Multidisciplinary Research Partnership: The integrative neuroscience of behavioral control), IBBT, the Research Foundation Flanders (FWO, Belgium) and the National Institutes of Health under Grant R01-CA113941

K. Van Audenhaege, R. Van Holen, K. Deprez and S. Vandenberghe are with Department of Electronics and Information Systems, MEDISIP, Ghent University-IBBT-IBiTech, De Pintelaan 185 block B, B-9000 Ghent, Belgium

R. Van Holen is supported by a postdoctoral fellowship of the Research Foundation Flanders (FWO)

J. Karp is with the Department of Radiology and the Department of Physics and Astronomy, University of Pennsylvania, USA.

S. Metzler is with the Department of Radiology, University of Pennsylvania, USA.

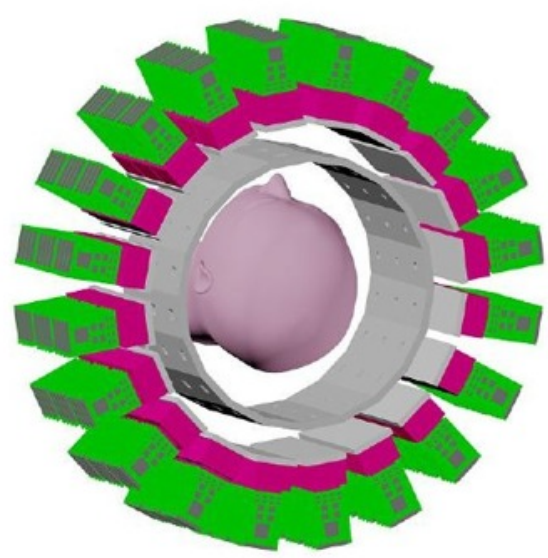

Fig. 1. Multi-pinhole brain SPECT collimator for a full-ring of gamma detector modules.

Tomography (PET), where a resolution of $2-6 \mathrm{~mm}$ can be achieved. PET tracers, however, have many drawbacks. The tracers have a shorter half-life, which makes them more difficult to handle and not suitable for long studies. Some PET isotopes even require a cyclotron at site. SPECT isotopes, on the other hand, have a longer half-life and therefore easier to handle.

In the past, simulations have shown that the resolution of brain SPECT can be improved using multi-pinhole collimators [3][4]. A better resolution results in a better diagnosis and thus in a better treatment for the patient. We designed a static full-ring multi-pinhole collimator for brain SPECT imaging with a target resolution of $4 \mathrm{~mm}$. The system is schematically represented in Figure 1. The collimator is optimized for an existing detector ring: the LaPET system [5] (a PET detector ring made of $24 \mathrm{LaBr} 3$ detectors of 27 by 60 pixels, each $4 \times 4 \times 30 \mathrm{~mm}$ large). The LaPET system was chosen because of its very good energy resolution (6\%) and its large (258 $\mathrm{mm}$ ) axial field-of-view (FOV).

In this paper we first describe the general design of the collimator. Next, we describe how the collimator is optimized to maximize the system performance. Finally, we describe the reconstruction of a resolution phantom simulated with a Monte-Carlo algorithm. 


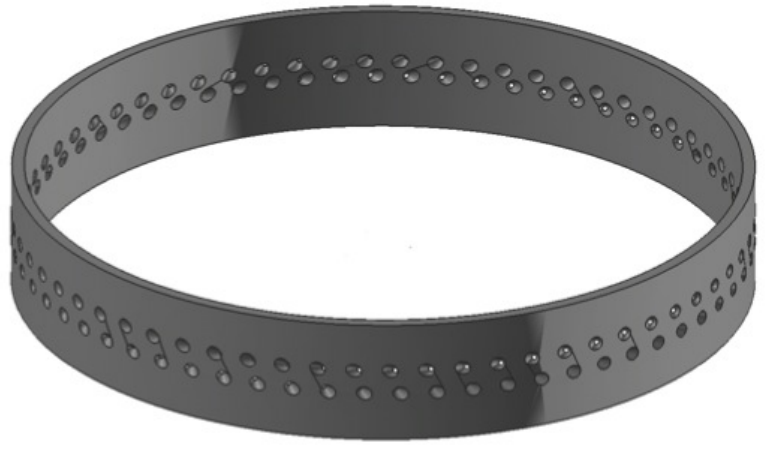

Fig. 2. The collimator

\section{MAterials AND METHODS}

\section{A. General design}

A naive design of a multi-pinhole collimator for brain SPECT would be a tungsten ring with one row of pinholes, where each pinhole sees the full transaxial FOV. However, this design has two issues. The first issue is the opening angle of the pinholes, which needs to be large enough to image the full transaxial FOV. A large opening angle, however, results in a large amount of pinhole penetration, which degrades the system resolution. The second issue is the angular sampling. In order to reconstruct the image, it is necessary to acquire projections from different angles. In a static full-ring multipinhole collimator, the number of projections is defined by the number of pinholes on the collimator ring, which is limited by the number of projections that fit on the detector without causing overlap. If this is not enough for complete angular sampling, the collimator is typically rotated. Rotating a heavy collimator, however, often induces geometric uncertainties that cause artifacts in the reconstructed image. In the following two paragraphs we describe how both issues are solved.

1) truncating pinholes: To reduce the opening angle, pinholes with truncated projections are used. Each pinhole sees only half the transaxial FOV. To guarantee transaxial sampling completeness, a second row of pinholes is used, which see the other half of the transaxial FOV. The two rows are spaced 8.12 $\mathrm{mm}$ apart (fig 2). The pinholes in the inferior row project on the inferior part of the detector and the pinholes in the superior row project on the superior part of the detector (fig 3). An annular slat is placed between the two pinhole-rings to prevent the projections from overlapping in the axial direction.

2) Shutter mechanism: To guarantee sufficient angular sampling, the collimator is equipped with collimating shutters that block all radiation when they are moved in front of a pinhole. This allows to include more pinholes on the collimator, without causing overlap. A limited amount of pinholes is selected to obtain a first set of projections. After a certain acquisition time, these pinholes are closed and the neighboring pinholes are opened. This process can be repeated multiple times. A sequence of shutter movements is thus performed to obtain an acquisition setup that simulates a rotational movement. Each

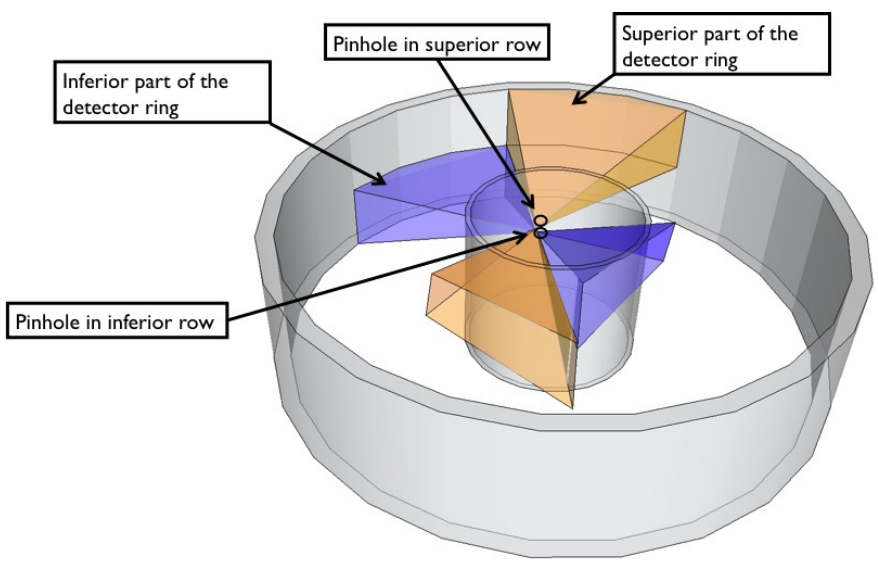

Fig. 3. FOV of two complementary pinholes

shutter also has a collimating element that is used to cut off the neighboring pinhole projections in order to obtain rectangular projections [6].

\section{B. Optimization}

In the previous section, we described the general design of the multi-pinhole collimator. In this section, we will describe how the collimator was optimized to maximize the system performance. The detector radius, the intrinsic spatial resolution of the detector and the FOV are known variables of the system and overlap is not allowed. The detector radius $D$ is 466.65 $\mathrm{mm}$, the intrinsic resolution $R_{i}$ is $4 \mathrm{~mm}$ and the FOV for brain imaging is modeled as a cylinder with a diameter of $220 \mathrm{~mm}$ and a length of $124 \mathrm{~mm}$ [4]. The collimator radius $c$ and the pinhole diameter $d$ on the other hand, are unknown and need to be optimized.

We aim at an overall image resolution of $4 \mathrm{~mm}$ but we chose to design the collimator to have a spatial resolution of $3.5 \mathrm{~mm}$ in the center of the field-of-view (CFOV). The optimization process now consists of maximizing the sensitivity given this spatial resolution $R_{C F O V}$. This is achieved by calculating the volume sensitivity as a function of the collimator radius, plotting the result and finding the maximum. The sensitivity $g$ of one pinhole for a certain point in the FOV, is described by equation (1). The volume sensitivity is then defined as an average of the sensitivity $g$ for each point in the FOV, multiplied by the number of pinholes $N$ that can simultaneously be opened without causing overlap. A ray-tracer is used to determine which points of the FOV are seen by the pinhole. For those points that are not seen by the pinhole, the sensitivity $g$ is zero. For the other points in the FOV, the sensitivity $g$ is described by the following equation [7]:

$$
g=\frac{d_{\text {Seff }}^{2} \cdot \sin ^{3} \theta}{16 b^{2}}
$$

$\theta$ is the angle of incidence measured from the plane of the pinhole, $b$ is the perpendicular distance from the point in the FOV to the plane of the pinhole and $d_{S e f f}$ is the sensitivity effective pinhole diameter, which is the physical pinhole diameter $d$, corrected for pinhole penetration. $d_{S e f f}$ is described by the following equation [8]: 


$$
d_{\text {Seff }}=\sqrt{d\left(d+\frac{2}{\mu} \tan \frac{\alpha}{2}\right)+\frac{2}{\mu^{2}} \tan ^{2} \frac{\alpha}{2}}
$$

$\alpha$ is the opening angle of the pinhole and $\mu$ is the attenuation coefficient of the collimator material. The value for tungsten used here is 3.3976 .

Thus, the sensitivity $g$ only depends on the pinhole diameter $d$. The volume sensitivity also depends on the number of pinholes $N$ and the FOV of the pinholes. Later in this section, it is shown that the pinhole diameter $d$ can be expressed as a function of the collimator radius $c$, given the spatial resolution $R_{C F O V}$. The number of pinholes $N$ that can simultaneously be opened without causing overlap can also be expressed as a function of the collimator radius. And for a given collimator radius, the FOV of the pinholes can also be determined. Thus, the volume sensitivity only depends on the collimator radius. By plotting the volume sensitivity as a function of the collimator radius, the optimum can easily be found.

In the remainder of this section we will describe how $d$ and $N$ can be calculated.

As the spatial resolution $R_{C F O V}$ is known, the resolution effective diameter $d_{R e f f}$ can be calculated using the following equation [9]:

$$
R_{C F O V}=\sqrt{\frac{R_{i}^{2}}{M^{2}}+\left(d_{\text {Reff }}\left(1+\frac{1}{M}\right)\right)^{2}}
$$

$M$ is the magnification of the system and is described by:

$$
M=\frac{D-c}{c}
$$

$d_{\text {Reff }}$ is the resolution effective pinhole diameter and is described by the following equations [10]:

$$
\begin{gathered}
d_{r e / /}=d+\frac{\ln 2}{\mu}\left(\tan ^{2} \frac{\alpha}{2}-\cot ^{2} \theta\right) \cot \frac{\alpha}{2} \sin \theta \\
d_{r e \perp}=\sqrt{\left(d+\frac{\ln 2}{\mu} \tan \frac{\alpha}{2} \sin \theta\right)^{2}-\left(\frac{\ln 2}{\mu}\right)^{2} \cos ^{2} \theta}
\end{gathered}
$$

$d_{r e / /}$ and $d_{r e \perp}$ are the resolution effective pinhole diameter in the parallel and the perpendicular direction. If the plane $X=0$ is defined by the normal to the detector plane and the vector from the pinhole to the voxel, then the parallel direction is along the $\mathrm{y}$ axis and the perpendicular direction is along the $\mathrm{x}$ axis. For the optimization process, the pinholes are assumed to focus on the CFOV, so the incidence angle $\theta$ is assumed to be $\frac{\pi}{2}$. Equations (5) and (6) can then be reduced to one equation:

$$
d_{\text {Reff }}=d+\frac{\ln 2}{\mu} \tan \frac{\alpha}{2}
$$

Equation (3), (4) and (7) are then combined and rearranged to explicitly show $d$ :

$$
d=\frac{\sqrt{(D-c)^{2} \cdot R_{C F O V}^{2}-R_{i}^{2}}}{D}-\frac{\ln 2}{\mu} \tan \frac{\alpha}{2}
$$

Finally, the number of pinholes $N$ that can simultaneously be opened is described by the following equation:

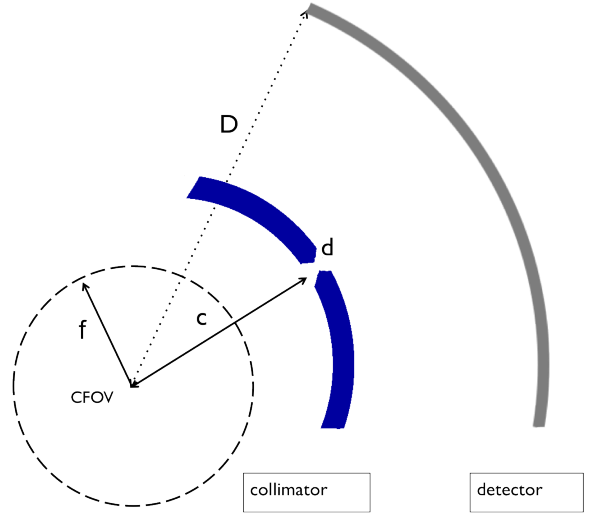

Fig. 4. Schematic overview of the design parameters.

$$
N=\frac{2 \pi}{\operatorname{acos}\left(\frac{f}{D}\right)-\operatorname{acos}\left(\frac{f}{c}\right)}
$$

with $f$ the radius of the FOV (110 mm) (fig 4).

The result of the optimization process is discussed in section III-A.

\section{System evaluation}

After optimizing the collimator, a Monte-Carlo simulation of a resolution phantom is performed using GATE [11]. The phantom is modeled using a grid with $1 \times 1 \times 1 \mathrm{~mm}$ voxels (fig $7 \mathrm{a}$. The phantom has a diameter of $220 \mathrm{~mm}$ and an axial length of $124 \mathrm{~mm}$. The rods have different diameters (from $1.5 \mathrm{~mm}$ to $8.0 \mathrm{~mm}$ ) and have an activity concentration that is 4 times higher than the background activity. Since we are only interested in the system resolution, a total activity of 30 $\mathrm{mCi}$ is used and phantom scatter and attenuation were not included in the simulation. 3 bed positions with an $8 \mathrm{~mm}$ shift are acquired in the central part of the axial FOV. The total duration of the simulated scan is 360 seconds. The projections are then reconstructed to a grid with $2 \times 2 \times 2 \mathrm{~mm}$ voxels using Ordered Subset Expectation Maximization (OSEM). The forward projector and the back projector are implemented using a ray-tracing algorithm. Resolution recovery is included, based on a multi-ray approach using 7 weighted rays [12]. The reconstruction was performed using 8 subsets and 20 iterations.

\section{RESUlTS AND DiscussiON}

\section{A. Optimal collimator design}

From equation (1) it follows that the smaller the collimator radius the higher the sensitivity in each point in the FOV. But the volume sensitivity also depends on the number of pinholes and the FOV of the pinholes. However, the smaller the collimator radius, the shorter the axial FOV and the lower the number of pinholes that can be opened simultaneously. Therefore, finding the optimal collimator radius is not trivial and the result strongly depends on the application. The optimal collimator radius is the one that maximizes the volume sensitivity, summed over the number of pinholes. 


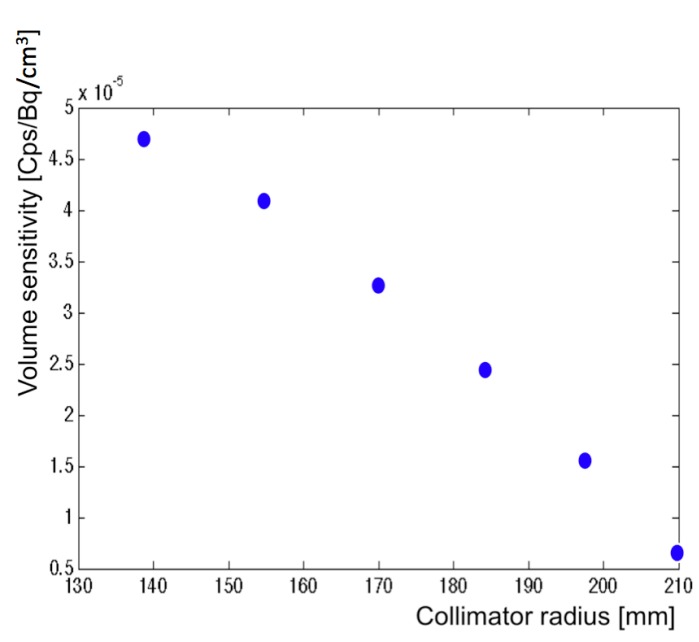

Fig. 5. Volume sensitivity as a function of the collimator radius
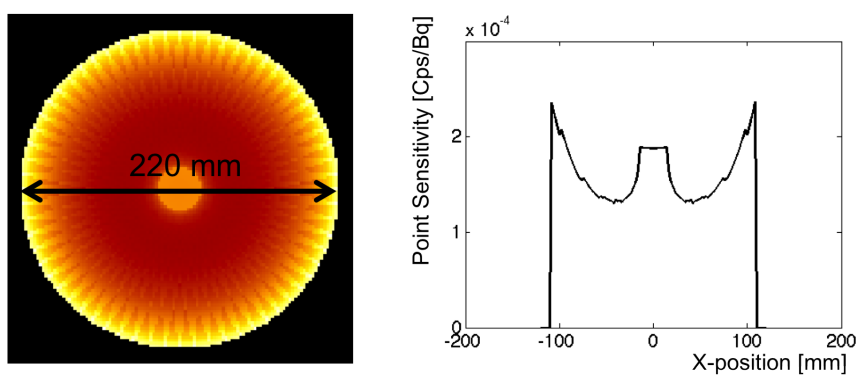

Fig. 6. Sensitivity map of the final design.

The result of the optimization process is shown in figure 5 . The volume sensitivity is plotted against the collimator radius, which has a lower and an upper limit. The lower limit is due to the physical size of the patient's head, which needs to fit in the collimator ring and the upper limit radius is due to the specification of $3.5 \mathrm{~mm}$ resolution in the CFOV. When the collimator radius increases, the magnification becomes smaller and at a certain point, the resolution of $3.5 \mathrm{~mm}$ cannot be achieved any more (even with very small pinholes). From figure 5 we can conclude that for this application, the larger axial FOV and the higher number of pinholes do not compensate for the decrease in point sensitivity and that the smallest collimator radius results in the highest volume sensitivity.

Based on this conclusion, we decided to choose the collimator radius as small as possible, while leaving enough space for the patient's head. We chose a collimator radius of 145 $\mathrm{mm}$ with 128 pinholes (64 in each row). 16 pinholes can then be opened simultaneously without causing overlap ( 8 in each row). For this collimator radius, a pinhole diameter of $2 \mathrm{~mm}$ is required to achieve a resolution of $3.5 \mathrm{~mm}$ in the CFOV. In the final design, each pinhole sees slightly more than half the transaxial FOV, so that sensitivity is higher in the central part of the FOV. Figure 6 shows a map of the sensitivity in each point of the FOV (for one slice). The sensitivity is highest in the CFOV $\left(1.84 .10^{-4} \mathrm{cps} / \mathrm{Bq}\right)$. The volume sensitivity is 4.35.10 ${ }^{-5} \mathrm{cps} / \mathrm{Bq} / \mathrm{cm}^{3}$.
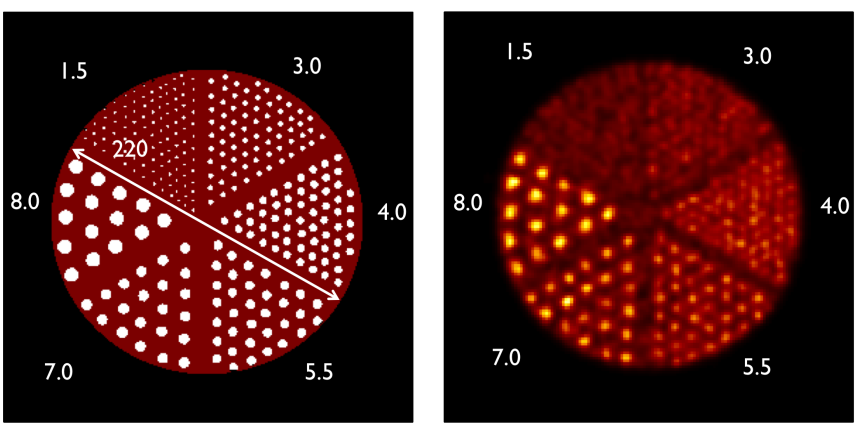

Fig. 7. (a) The resolution phantom and (b) a reconstruction.

After optimizing the collimator, the final design is validated using Mont-Carlo simulations. The projection data of a resolution phantom is successfully reconstructed (fig $7 \mathrm{~b}$ ) and the resulting image shows that a resolution of $4 \mathrm{~mm}$ is indeed achieved

\section{CONCLUSION}

We have presented a non-rotating multi-pinhole collimator for brain SPECT. Simulations and calculations predict a system resolution of $4 \mathrm{~mm}$, a sensitivity of $1.84 .10^{-4} \mathrm{cps} / \mathrm{Bq}$ in the CFOV and a volume sensitivity of $4.35 .10^{-5} \mathrm{cps} / \mathrm{Bq} / \mathrm{cm}^{3}$. The point sensitivity is comparable to the sensitivity of a dualhead system with LEHR parallel-beam collimators but the volume sensitivity is 4 times lower. However, the resolution is 2 times better, a trade-off that is supported by Muehllehner [1]. A quantitative study based on CNR-measurements will also be performed to further investigate the resulting image quality. In a next phase, multiplexing will be investigated using different configurations of the shutters on the prototype. Also, a prototype of this collimator will be tested on the LaPET system. This will allow us to determine the potential to leverage the LaPET scanner for SPECT imaging.

\section{REFERENCES}

[1] G. Muehllehner et al., "Effect of resolution improvement on required count density in ECT imaging: a computer simulation," Physics in medicine and biology, vol. 2, 1985.

[2] D. G. Amen, M. Trujillo, A. Newberg, K. Willeumier, R. Tarzwell, J. C. Wu, and B. Chaitin, "Brain SPECT Imaging in Complex Psychiatric Cases : An Evidence-Based, Underutilized Tool," The Open Neuroimaging Journal, vol. 5, pp. 40-48, 2011.

[3] M. Rogulski, H. Barber, H. Barrett, R. Shoemaker, and J. Woolfenden, "Ultra-high-resolution brain SPECT imaging: simulation results," IEEE Transactions on Nuclear Science, vol. 40, no. 4, pp. 1123-1129, 1993.

[4] M. Goorden, M. Rentmeester, and F. Beekman, "Theoretical analysis of full-ring multi-pinhole brain SPECT," Phys. Med. Biol, vol. 54, no. 21, pp. 6593-6610, 2009

[5] M. Daube-witherspoon, S. Surti, A. Perkins, C. Kyba, M. Werner, S. Matej, and J. Karp, "Imaging Performance of a LaBr3-Based Timeof-Flight PET Scanner," IEEE Nuclear Science Symposium Conference Record, pp. 5463-5467, 2008.

[6] K. Van Audenhaege, K. Deprez, S. Vandenberghe, and R. Vanholen, "Collimating shutter," Application for European Patent 11165483.6, May 10th, 2011.

[7] J. R. Mallard and M. J. Myers, "The Performance of a Gamma Camera for the Visualization of Radioactive Isotopes in vivo," Physics in Medicine and Biology, vol. 8, no. 2, pp. 165-182, Jun. 1963.

[8] D. Paix, "Pinhole imaging of gamma rays," Phys. Med. Biol., no. 12, p. $489500,1967$. 
[9] H. Anger, "Radioisotope cameras Instrumentation," Nuclear Medicine, no. 1, p. 485552, 1967.

[10] R. Accorsi and S. D. Metzler, "Analytic determination of the resolutionequivalent effective diameter of a pinhole collimator." IEEE transactions on medical imaging, vol. 23, no. 6, pp. 750-63, Jun. 2004.

[11] S. Jan et al., "GATE: a simulation toolkit for PET and SPECT," Phys. Med. Biol., vol. 49, pp. 4543-4561, Oct. 2004.

[12] C. Vanhove, A. Andreyev, M. Defrise, J. Nuyts, and A. Bossuyt, "Resolution recovery in pinhole SPECT based on multi-ray projections: a phantom study." European journal of nuclear medicine and molecular imaging, vol. 34, no. 2, pp. 170-80, Feb. 2007. 